\title{
Evidence for No Significant Impact of Müllerian Anomalies on Reproductive Outcomes of Twin Pregnancy in Korean Women
}

\author{
Sohyun Shim, ${ }^{1}$ Yoon-Mi Hur, ${ }^{2}$ Da Hee Kim, ${ }^{1}$ Seok Ju Seong, ${ }^{1}$ Mi-La Kim, ${ }^{1}$ and Joong Sik Shin ${ }^{1}$ \\ ${ }^{1}$ Department of Obstetrics and Gynecology, CHA Gangnam Medical Center, CHA University, Seoul, South Korea \\ ${ }^{2}$ Department of Education, Mokpo National University, Jeonnam, South Korea
}

\begin{abstract}
The present article aimed to evaluate the impact of congenital Müllerian anomalies (MA) on twin pregnancy after 24 gestational weeks in Korean women. All records of twin pregnancies in a large maternity hospital in Korea between January 2005 and July 2013 were analyzed. Patients with monochorionic monoamniotic (MCMA) twins, non-Korean patients, patients with twins delivered prior to 24 gestational weeks, and patients with miscarriage of one fetus or intrauterine fetal death (IUFD) before 24 gestational weeks were excluded from data analysis. In total, 1,422 women with twin pregnancy were eligible for data analysis, including 17 (1.2\%) who had a known congenital MA (septate uterus, bicornuate uterus, arcuate uterus, and unicornuate uterus). Except for the mode of conception, baseline demographics were similar between women with MA and those without MA. No significant differences were found in pregnancy outcomes of gestational age at delivery $(p=.86)$, birth weight of smaller and larger twins $(p=.54$ and $p=.65)$, and number of twins with birth weight $<5$ th percentile for gestational age $(p=.43)$. The rates of obstetrical complications such as pre-eclampsia, gestational diabetes mellitus (GDM), placenta previa, cerclage, IUFD, and postpartum hemorrhage were not significantly different between the two groups either. We concluded that the presence of congenital MA may not increase obstetrical risks in outcomes of pregnancy of twins delivered after 24 gestational weeks.
\end{abstract}

Keywords: Müllerian anomalies, twin, pregnancy outcome, obstetrical complications, uterine anomalies

Congenital MA of the female reproductive tract comprise a range of abnormalities caused by failure of any part of the complex developmental series of the female reproductive tracts, including failure of Müllerian duct development, elongation, fusion, canalization, and septal resorption. In MA, cervix, fallopian tubes, vagina, and uterine anomalies are most common. The prevalence of MA in the general population is unknown, but several recent studies indicated that MA occurs in approximately $1-4 \%$ of reproductive-aged women, and $2-8 \%$ of infertile women (Chan et al., 2011; Lin et al., 2002; Propst \& Hill, 2000; Simon et al., 1991). Although some patients have no significant clinical symptoms, congenital MA is associated with infertility and/or adverse pregnancy outcomes: approximately $5-10 \%$ of women with MA have been documented to have experienced recurrent early pregnancy loss and up to $25 \%$ of women have experienced late first- or second-trimester pregnancy loss, preterm labor, or fetal malpresentation following cesarean delivery (Lin et al., 2002; Propst \& Hill, 2000; Simon et al., 1991).
A meta-analysis based on nine studies consisting of 3,805 patients with MA (Chan et al., 2011) evaluated the effects of three types of MA (arcuate uteri, canalization defects, and unification defects) on reproductive outcomes. The meta-analysis found that while all three types were significantly related to fetal malformation at delivery, arcuate uteri was specifically associated with increased rates of second-trimester miscarriage, canalization defects, and with reduced pregnancy rates, increased rates of firsttrimester miscarriage, and preterm birth, and unification defects as associated with increased rates of preterm delivery. However, all of the cases included in the meta-analysis

RECEIVED 1 November 2015; ACCEPTED 13 January 2016. First published online 16 February 2016.

ADDRESS FOR CORRESPONDENCE: Joong Sik Shin or Mi-La Kim, Department of Obstetrics and Gynecology, CHA Gangnam Medical Center, CHA University, 566, Nonhyeon-ro, Gangnamgu, Seoul 135-081, South Korea. E-mail: shinjs@cha.ac.kr; mila76@naver.com 
were about singleton pregnancy, and none about twin pregnancies. Studies of the impact of MA on twin pregnancies were rarely done. To the best of our knowledge, only 14 case reports (Ahmad et al., 2000; Arora et al., 2007; Aruh et al., 2005; Brown, 1999; Check et al., 1992; Cruceyra et al., 2011; Doruk et al., 2013; Kekkonen et al., 1991; NahraLynch \& Toffle, 1997; Nahum, 1997; Nanda et al., 2009; Nohara et al., 2003; Sugaya, 2010; Weissman et al., 2006), and one comparative study (Fox et al., 2014) examined the effect of MA on twin pregnancies to date. These studies also suggested that the presence of MA in twin pregnancies significantly increased the risks for negative reproductive outcomes, including cerclage, preterm birth, and lower birth weights and other obstetrical complications.

Due to a recent rapid development of various assisted reproductive technologies (ART), the rates of twin births have been increased dramatically worldwide. For example, the twinning rates rose from 18.9 in 1980 to 33.7 per thousand live births in 2013 in the United States (Martin et al., 2015). In South Korea, twin births increased from approximately 10 in 1981 to 18.8 per thousand births in 2007 (Hur \& Kwon, 2005; Hur \& Song, 2009). As clinicians generally recommend ART to women with congenital MA who experience infertility, the incidence of twin pregnancies in women with MA is expected to increase in the future. However, twin pregnancy itself is well known to have increased risks in obstetrical complications, such as pre-eclampsia (Krotz et al., 2002), and negative outcomes of pregnancy such as preterm delivery (Kogan et al., 2002) and low birth weight (Martin et al., 2015). Thus, it is important to investigate whether or not the presence of MA confers additional risks on twin pregnancies. In this study, we reviewed all records of twin pregnancies in a large maternity hospital in Korea between January of 2005 and July of 2013 and compared obstetrical outcomes and complication rates in twin pregnancies with and without congenital MA.

\section{Materials and Methods}

In a review of maternity records, we used the following inclusion criteria for data analysis: (1) twins delivered after 24 gestational weeks; (2) initially triplet pregnancy reduced to twin pregnancy at the time of delivery due to miscarriage of one fetus, IUFD, or selective abortion before 24 gestational weeks; (3) one or two fetus IUFD after 24 gestational weeks. We excluded the following cases from analysis: (1) patients with MCMA twins because of the risk of cord entanglement and associated risk of IUFD or intended preterm delivery; (2) non-Korean patients; (3) patients with missed abortion of one fetus or IUFD less than 24 gestational weeks.

In our study, all of the congenital MA were diagnosed before pregnancy. We classified MA according to the American Fertility Society (1988) classification, using the combinations of at least two diagnostic tools: transvaginal ultrasonography (TVS), three-dimensional ultrasonogra- phy (3-D ultrasonography), hysterosalpingography (HSG), hysteroscopy, laparoscopy, and magnetic resonance imaging (MRI). We included the corrected anomalies such as complete or partial resection of uterine septum using hysteroscopy in the congenital MA group (AFS classifications, 1988).

All twin pregnancies were managed following the uniform protocol of our clinic. During the first trimester, we confirmed the gestational age and chorionicity by TVS. If the patient visited our hospital after late second trimester and chorionicity was unclear, we confirmed the chorionicity by pathological examination of the placenta. We adapted the definition of birth weight percentiles for gestational age presented in standard tables for twin pregnancies (Yarkoni et al., 1987).

Statistical analysis was performed using SPSS (version 12.0; SPSS, Chicago, IL, USA). Chi-square and Fisher's exact tests were used for analysis of categorical variables. Quantitative variables were compared by means of Mann-Whitney $\mathrm{U}$ tests. A $p$ value of $<.05$ was considered statistically significant.

\section{Results}

During the study period, a total of 1,478 twin deliveries after gestational age of 24 weeks were recorded. Of these, 5 cases were excluded as they had MCMA twins, 36 cases were patients who were non-Korean, and 13 cases were patients who had experienced miscarriage of one fetus or two fetuses or IUFD at less than 24 gestational weeks. In total, 1,422 patients were analyzed for the purpose of the present study. Seventeen cases $(1.2 \%)$ had a known congenital MA (septate uterus; $n=10)$, bicornuate uterus $(n=3)$, arcuate uterus $(n=2)$, and unicornuate uterus $(n=2)$. All of the patients diagnosed with a septated uterus underwent hysteroscopic septotomy before the twin pregnancy.

The baseline characteristics of the patients with MA and those without MA are compared in Table 1. Only mode of conception was significantly different between patients with MA and those without MA $(p<.001)$. However, a post-hoc analysis indicated that the rate of in vitro fertilization (IVF) was not significantly different between the two groups ( $64.7 \%$ vs. $71.8 \%$; $p=59)$, while two other categories (natural or timed intercourse [TI], controlled ovarian hyperstimulation $[\mathrm{COH}]+\mathrm{TI}$; intrauterine insemination [IUI] or $\mathrm{COH}+\mathrm{IUI}$ ) showed significant differences.

The pregnancy outcomes between the two groups are shown in Table 2. There were no significant differences in the gestational age at delivery, birth weight of the twins, birth weight discordancy, or percentage of twins with birth weight in the $<5$ th percentile for gestational age. Table 3 compares the rates of obstetric complications between the two groups. No statistically significant differences were observed in the rates of pre-eclampsia, GDM, placenta previa, 
TABLE 1

Baseline Characteristics of Patients Who Gave Birth to Twins in a Large Maternity Hospital in Korea Between January 2005 and July 2013

\begin{tabular}{|c|c|c|c|}
\hline & Müllerian anomalies $(n=17)$ & No Müllerian anomalies $(n=1405)$ & $p$ value \\
\hline Mean (SD, median, range) of maternal age in years ${ }^{a}$ & $33.0 \pm 3.3(33,28-39)$ & $34.6 \pm 3.5(35,23-52)$ & .06 \\
\hline Number $(\%)$ of nulliparity ${ }^{b}$ & $14(82.4 \%)$ & $1,168(83.1 \%)$ & 1.00 \\
\hline $\begin{array}{l}\text { Mean (SD, median, range) of pre-pregnancy BMI }(\mathrm{kg} / \mathrm{m} 2)^{a} \\
\text { Number }(\%) \text { of mode of conception }\end{array}$ & $21.0 \pm 2.3(20.5,18.4-26.6)$ & $21.5 \pm 3.1(20.8,14.3-39)$ & .62 \\
\hline Natural or $\mathrm{TI}, \mathrm{COH}+\mathrm{TI}$ & $1(5.9 \%)$ & $316(22.5 \%)$ & $<.001$ \\
\hline $\mathrm{IUI}$ or $\mathrm{COH}+\mathrm{IUI}$ & $5(29.4 \%)$ & $80(5.7 \%)$ & \\
\hline IVF & $11(64.7 \%)$ & $1,009(71.8 \%)$ & \\
\hline Number (\%) chorionicity ${ }^{b}$ & & & \\
\hline Monochorionic & $0(0 \%)$ & $103(7.3 \%)$ & .63 \\
\hline Dichorionic & $17(100 \%)$ & $1,302(92.7 \%)$ & \\
\hline
\end{tabular}

Note: $\mathrm{BMI}=$ body mass index; $\mathrm{TI}=$ timed intercourse $\mathrm{COH}=$ controlled ovarian hyperstimulation; IUI = intrauterine insemination; IVF = in vitro fertilization.

a Mann-Whitney U

${ }^{b}$ Fisher exact test.

cchi-square.

\section{TABLE 2}

Comparisons of Twin Pregnancy Outcomes in Patients With Müllerian Anomalies (MA) and Without MA

\begin{tabular}{|c|c|c|c|}
\hline & $\mathrm{MA}(n=17)$ & No MA $(n=1,405)$ & $p$ value \\
\hline GA at delivery in weeks ${ }^{a}$ & $35.5 \pm 3.2(37,26.3-38.1)$ & $36.1 \pm 2.2(37,24.6-40)$ & .86 \\
\hline \multicolumn{4}{|l|}{ Delivery in weeks ${ }^{b}$} \\
\hline $\mathrm{GA}$ at 24 weeks $-<28$ weeks & $1(5.9 \%)$ & $26(1.9 \%)$ & .86 \\
\hline GA at 28 weeks $-<32$ weeks & $1(5.9 \%)$ & $51(3.6 \%)$ & \\
\hline $\mathrm{GA}$ at 32 weeks $-<34$ weeks & $1(5.9 \%)$ & $89(6.3 \%)$ & \\
\hline GA at 34 weeks $-<37$ weeks & $5(29.4 \%)$ & $534(38.0 \%)$ & \\
\hline $\mathrm{GA} \geq 37$ weeks & $9(52.9 \%)$ & $705(50.2 \%)$ & \\
\hline Mean (SD, median, range) of birth weight of larger twin ${ }^{a}$ & $2,491.8 \pm 655.2(2670,900-3210)$ & $2,552.9 \pm 453.3(2615,710-3940)$ & .65 \\
\hline Mean (SD, median, range) of birth weight of smaller twin & $2,197.7 \pm 684.1(2470,870-2970)$ & $2,255.8 \pm 455(2320,140-3700)$ & .54 \\
\hline Number of twin birth weight $<5$ th\% ${ }^{c}$ & $3(17.6 \%)$ & $391(27.8 \%)$ & .43 \\
\hline Mean (SD, median, range) of birth weight ${ }^{a}$ & $4,689.4 \pm 1321.8(4940,2530-6970)$ & $4,808.7 \pm 867.0(4930,1240-7620)$ & .55 \\
\hline Mean (SD, median, range) of birth weight difference ${ }^{a}$ & $274.1 \pm 217.9(330,70-770)$ & $297.9 \pm 269.6(230,0-3100)$ & .79 \\
\hline
\end{tabular}

Note: $\mathrm{GA}=$ gestational age; sum of birth weight= sum of birth weight of two members of a twin pair; birth weight difference $=$ birth weight difference between two members of a twin pair.

a Mann-Whitney U.

b Likelihood ratio test.

'Fisher's exact test.

\section{TABLE 3}

The Rates of Obstetrical Complications of Twin Pregnancy in Patients With Müllerian Anomalies (MA) and Those Without MA

\begin{tabular}{llrr}
\hline Obstetrical complications & MA $(n=17)$ & No MA $(n=1405)$ & p value \\
\hline Pre-eclampsia $^{a}$ & $3(17.6 \%)$ & $104(7.4 \%)$ & .13 \\
GDM $^{a}$ & $0(0 \%)$ & $50(3.6 \%)$ & 1.00 \\
Placenta previa $^{a}$ & $1(5.9 \%)$ & $37(2.6 \%)$ & .37 \\
Cerclage $^{a}$ & $1(5.9 \%)$ & $60(4.3 \%)$ & .53 \\
P24 weeks IUFD $^{a}$ & $0(0 \%)$ & $9(0.6 \%)$ & 1.00 \\
Postpartum hemorrhage $^{a}$ & $4(23.5 \%)$ & $139(9.9 \%)$ & .08 \\
\hline
\end{tabular}

Note: Values are presented as number (\%). GDM = gestational diabetes mellitus; IUFD = intrauterine fetal death.

${ }^{\text {a }}$ Fisher exact test.

cervical cerclage due to short cervix, IUFD after 24 gestational weeks, or postpartum hemorrhage.

\section{Discussion}

Obstetric complications of MA were mainly due to the decreased space of the uterus, inadequate musculature, impaired distension ability during the pregnancy, abnormal myometrial and cervical function, and abnormal endometrial development that can interrupt normal early embryo development after implantation (Acien, 1993). In twin pregnancies, these anatomic and functional defects can result in more serious negative outcomes (Braun et al., 2005; Kim et al., 2008; Leible et al., 1998). However, our study showed that the presence of MA does not significantly increase the risks for negative outcomes or complications in twin pregnancy as long as twins survive until 24 gestational weeks, suggesting that the impact of MA may be minimal once twin fetuses reach the limit of viability.

Table 4 lists cases from all studies of twin pregnancy with MA published in English to date, which included 48 cases, consisting of 14 cases from the case reports, 17 cases from Fox et al. (2014), and 17 cases from our study. For the purpose of comparison, the list only included twins delivered after 24 gestational weeks. Although the Fox et al. study (2014) in the list is the only comparative study similar to ours, the results of the Fox et al. study are not consistent with ours. Fox et al. found that uterine anomalies were significantly associated with increased risks of cerclage $(p<$ 
TABLE 4

A Review of Papers Published in English on Complications and Outcomes of Twin Pregnancies in Patients With Müllerian Anomalies ${ }^{\mathrm{a}}$

\begin{tabular}{|c|c|c|c|c|c|c|}
\hline Study & $\mathrm{G} / \mathrm{P}$ & Mode of conception & Types of Müllerian anomalies & Delivery in weeks & Pregnancy outcome & Remark or complications \\
\hline Kekkonen et al. (1991) & $2 / 1$ & Natural pregnancy & Uterine didelphys & 38 wks & Different cavity: C/S F \& M & Unremarkable \\
\hline Check et al. (1992) & $1 / 0$ & T-ET & Unicornuate uterus & 35 wks & $\begin{array}{l}\text { NSVD Vx M/2400g, C/S d/t } \\
\text { cord prolapse } V \times \mathrm{M} / 1994\end{array}$ & Preterm labor \\
\hline Nahum (1997) & $1 / 0$ & Natural pregnancy & $\begin{array}{l}\text { Unicornuate uterus c } \\
\text { non-communicating } \\
\text { rudimentary horn }\end{array}$ & $\begin{array}{c}\text { 1st: } 28+2 \text { wks } 2 \text { nd: } \\
29+3 \text { wks }\end{array}$ & $\begin{array}{l}\text { 1st: Uterine cavity } \mathrm{C} / \mathrm{S} \mathrm{d} / \mathrm{t} \\
\text { breech, intractable preterm } \\
\text { labor } \mathrm{F} / 971 \mathrm{~g} 2 \mathrm{nd} \text { : } \\
\text { rudimentary horn, NSVD d/t } \\
\text { rudimentary horn rupture } \mathrm{Vx} \\
\mathrm{F} / 1120 \mathrm{~g}\end{array}$ & $\begin{array}{l}\text { 1st C/S: ipsilateral hypogastric } \\
\text { and ovarian artery ligation to } \\
\text { arrest myometrial } \\
\text { hemorrhage 2nd NSVD: } 8 \\
\text { days later }\end{array}$ \\
\hline Nahra-Lynch \& Toffle (1997) & $4 / 1$ & $\mathrm{COH}+\mathrm{IUI}$ & Unicornuate uterus & $34+3$ wks & $\mathrm{C} / \mathrm{S} ; \mathrm{Vx} 2370 \mathrm{~g}, \mathrm{Br} 1860 \mathrm{~g}$ & $\begin{array}{l}\text { Cervical incompetence, } \\
\text { preterm labor, postpartum } \\
\text { atony }\end{array}$ \\
\hline Brown (1999) & $10 / 6$ & Natural pregnancy & Uterine didelphys & 26 wks & $\begin{array}{l}\text { Different cavity: C/S Br F/900g, } \\
\mathrm{Br} \mathrm{M} / 800 \mathrm{~g}\end{array}$ & One uterus: Abruption placenta \\
\hline Ahmad et al. (2000) & $2 / 0$ & Natural pregnancy & Uterine didelphys & $31 \mathrm{wks}$ & $\mathrm{C} / \mathrm{S} F / 1480, \mathrm{~F} / 1421 \mathrm{gm}$ & $\begin{array}{l}\text { IUGR, elevated liver enzyme, } \\
\text { proteinuria }\end{array}$ \\
\hline Nohara et al. (2003) & $2 / 0$ & $\mathrm{CC}+\mathrm{IUI}$ & Uterine didelphys & $\begin{array}{l}\text { 1st: } 25 \text { wks 2nd: } 35 \\
\text { wks }\end{array}$ & $\begin{array}{l}\text { Different cavity: } 1 \mathrm{st} \mathrm{C} / \mathrm{S} \mathrm{d} / \mathrm{t} \\
\text { fetal distress, } \\
\text { chorioamnionitis M/764gm } \\
\text { 2nd NSVD d/t spontaneous } \\
\text { labor } \mathrm{M} / 2360 \mathrm{gm}\end{array}$ & $\begin{array}{l}\text { 1st baby: PPROM and } \\
\text { chorioamnionitis, subsequent } \\
\text { fetal distress } 2 \text { nd NSVD: } \\
\text { preterm labor ( } 66 \text { days later) }\end{array}$ \\
\hline Aruh et al. (2005) & $1 / 0$ & IVF-ET & Bicornuate uterus & 37 wks & $\begin{array}{l}\text { Separate horn: C/S M/2600g, } \\
\mathrm{M} / 2600 \mathrm{~g}\end{array}$ & Uneventful \\
\hline Weissman et al. (2006) & $1 / 0$ & IVF-ET & Complete septum & 34 wks & $\mathrm{F} / 1680 \mathrm{gm}, \mathrm{M} / 2035 \mathrm{gm}$ & $\begin{array}{l}\text { After } 25 \text { weeks, pre-term labor, } \\
\text { cervical shortening, PPROM }\end{array}$ \\
\hline Arora et al. (2007) & $2 / 1$ & Natural pregnancy & Bicornuate uterus & 37 wks & $\begin{array}{l}\text { Separate horn: C/S Vx, } \\
\mathrm{M} / 2300 \mathrm{~g}, \mathrm{Br} F / 2500 \mathrm{~g}\end{array}$ & Pre-eclampsia \\
\hline Nanda et al. (2009) & $1 / 0$ & Natural pregnancy & $\begin{array}{l}\text { Unicornuate uterus c } \\
\text { non-communicating } \\
\text { rudimentary horn }\end{array}$ & 35 wks & $\begin{array}{l}\mathrm{C} / \mathrm{S} \mathrm{d} / \mathrm{t} \text { uncontrolled high BP } \\
\text { 1st: Uterine cavity F/2700g, } \\
\text { 2nd: non-communicating } \\
\text { rudimentary horn F/1900g }\end{array}$ & $\begin{array}{l}\text { Pre-eclampsia, postpartum } \\
\text { hemorrhage, wound } \\
\text { dehiscence with sepsis }\end{array}$ \\
\hline Sugaya (2010) & $1 / 0$ & IUI & Unicornuate uterus & $36+1 \mathrm{wks}$ & $C / S: V \times 2002 g, V \times 2164 g m$ & $\begin{array}{l}\text { Prophylactic cervical cerclage, } \\
\text { preterm labor, shortening of } \\
\text { Cx length at } 22 \text { wks, PPROM }\end{array}$ \\
\hline Cruceyra et al. (2011) & $1 / 0$ & Natural pregnancy & Bicornuate uterus & 38 wks & $\begin{array}{l}\text { Separate horn: C/S Vx, } \\
M / 2910 \mathrm{~g}, \mathrm{Vx} M / 2680 \mathrm{~g}\end{array}$ & Uneventful \\
\hline Doruk et al. (2013) & Multi-parous & Natural pregnancy & Bicornuate uterus & 35 wks & $\begin{array}{l}\text { Separate horn: } C / S(d / t \\
\text { previous } C / S) V x 2140 g, V x \\
2270 g\end{array}$ & Uneventful \\
\hline \multirow[t]{5}{*}{ Fox et al. (2014) } & $2 / 1$ & NA & Unicornuate & 35 wks & $\mathrm{RC} / \mathrm{S}$ & GDM, preterm labor \\
\hline & $1 / 0$ & NA & Uterine septum & $32+5$ wks & NSVD & $\begin{array}{l}\text { Cervical incompetence, } \\
\text { PPROM at } 31 \text { wks }\end{array}$ \\
\hline & $2 / 0$ & NA & Uterine septum & $36+5$ wks & $\mathrm{C} / \mathrm{S}$ Breech & $\begin{array}{l}\text { Ultrasound indicated cervical } \\
\text { cerclage at } 20 \mathrm{wks} \text {, preterm } \\
\text { labor }\end{array}$ \\
\hline & $2 / 0$ & NA & Uterine septum & $36+4 \mathrm{wks}$ & NSVD & Cholestasis of pregnancy \\
\hline & $1 / 0$ & NA & Uterine septum & $25+1 \mathrm{wks}$ & $\begin{array}{l}\text { NSVD, neonatal death of both } \\
\text { twins }\end{array}$ & $\begin{array}{l}\text { Sub-chorionic hematoma, } \\
\text { preterm labor }\end{array}$ \\
\hline
\end{tabular}


TABLE 4

Continued

\begin{tabular}{|c|c|c|c|c|c|c|}
\hline Study & $\mathrm{G} / \mathrm{P}$ & Mode of conception & Types of Müllerian anomalies & Delivery in weeks & Pregnancy outcome & Remark or complications \\
\hline & $3 / 0$ & NA & Uterine septum & $37+2$ wks & $\mathrm{C} / \mathrm{S}$ Breech & Unremarkable \\
\hline & $2 / 1$ & NA & Uterine septum & $34+2 \mathrm{wks}$ & $\mathrm{RC} / \mathrm{S}$ & Prior cone biopsy, preterm labor \\
\hline & $2 / 0$ & NA & Uterine septum & $33+2 \mathrm{wks}$ & $\mathrm{C} / \mathrm{S}$ & $\begin{array}{l}\text { Chronic hypertension, superimposed } \\
\text { severe pre-eclampsia }\end{array}$ \\
\hline & $4 / 0$ & NA & Uterine septum & $33+6$ wks & $\mathrm{C} / \mathrm{S}$ Breech & $\begin{array}{l}\text { Multifetal reduction 4->2, severe } \\
\text { pre-eclampsia }\end{array}$ \\
\hline & $2 / 1$ & NA & Uterine septum & $36+6$ wks & $\mathrm{RC} / \mathrm{S}$ & Retained placenta \\
\hline & $4 / 0$ & IVF & Uterine didelphys & $32+6$ wks & Different cavity: $\mathrm{C} / \mathrm{S}$ vasa previa & $\begin{array}{l}\text { Ultrasound indicated cervical cerclage } \\
\text { at } 23 \text { wks, cholestasis of pregnancy, } \\
\text { preterm labor }\end{array}$ \\
\hline & $8 / 1$ & NA & Bicornuate uterus & $37+6 \mathrm{wks}$ & $\mathrm{C} / \mathrm{S}$ Breech & Unremarkable \\
\hline & $3 / 0$ & NA & Bicornuate uterus & $26+4$ wks & $\begin{array}{l}C / S \text {, IUFD of twin } A \text {, neonatal death in } \\
\text { twin } B\end{array}$ & Preterm labor, IUFD, neonatal death \\
\hline & $1 / 0$ & NA & Bicornuate uterus & $30+3$ wks & Same horn: C/S placenta abruption & $\begin{array}{l}\text { Spontaneous reduction of 4->2, } \\
\text { preterm labor }\end{array}$ \\
\hline & $1 / 0$ & NA & Arcuate uterus & $36+6$ wks & NSVD & Preterm labor \\
\hline & $5 / 0$ & NA & Arcuate uterus & $36+4$ wks & NSVD & $\begin{array}{l}\text { Multifetal reduction 3->2, preterm } \\
\text { labor }\end{array}$ \\
\hline & $4 / 1$ & NA & Arcuate uterus & $32+3$ wks & $\mathrm{C} / \mathrm{S}$ Breech & $\begin{array}{l}\text { History indicated cerclage, preterm } \\
\text { labor }\end{array}$ \\
\hline \multirow[t]{17}{*}{ Present study } & $1 / 0$ & $\mathrm{COH}+\mathrm{TI}$ & Arcuate uterus & 37 wks & $\mathrm{C} / \mathrm{S}$ Vx F/2670g, Br F/2510g & Unremarkable \\
\hline & $1 / 0$ & T-ET & Arcuate uterus & $37+5$ wks & $\mathrm{C} / \mathrm{S} \mathrm{Br} \mathrm{F} / 2630 \mathrm{~g}, \mathrm{Br} \mathrm{F} / 2880 \mathrm{~g}$ & Uterine atony \\
\hline & $1 / 0$ & IVF & Unicornuate uterus & $37+3$ wks & $\mathrm{C} / \mathrm{S} V \times \mathrm{F} / 2380 \mathrm{~g}, \mathrm{~V} \times \mathrm{M} / 2450 \mathrm{~g}$ & Unremarkable \\
\hline & $1 / 0$ & IVF & Unicornuate uterus & $34+4$ wks & $\mathrm{C} / \mathrm{S} V \times \mathrm{M} / 2470 \mathrm{~g}, \mathrm{~V} \times \mathrm{F} / 1690 \mathrm{~g}$ & Severe pre-eclampsia \\
\hline & $2 / 0$ & T-ET & Bicornuate uterus & $37+2$ wks & $\begin{array}{l}\text { Same horn: C/S Vx F/2640g, Vx } \\
\text { F/2780g }\end{array}$ & Unremarkable \\
\hline & $3 / 1$ & IVF & Bicornuate uterus & $35+2 \mathrm{wks}$ & $\begin{array}{l}\text { Same horn: C/S Br F/2560, Br } \\
M / 2470 \mathrm{~g}\end{array}$ & Preterm labor \\
\hline & $1 / 0$ & IVF & Bicornuate uterus & $37+3$ wks & $\begin{array}{l}\text { Same horn: C/S Br F/2420g, Br } \\
\text { F/2870g }\end{array}$ & Preterm labor \\
\hline & $1 / 0$ & $\mathrm{COH}+\mathrm{IUI}$ & Uterine septum & $37+2 \mathrm{wks}$ & $\begin{array}{l}\text { C/S placenta abruption, } \mathrm{Vx} F / 2540 \mathrm{~g} \text {, } \\
\mathrm{Br} \mathrm{M} / 3010 \mathrm{~g}\end{array}$ & Placenta abruption \\
\hline & $3 / 1$ & IUI & Uterine septum & $26+2$ wks & $\mathrm{C} / \mathrm{S} \mathrm{Br} \mathrm{F/900g}, \mathrm{V} \times \mathrm{M} / 870 \mathrm{~g}$ & $\begin{array}{l}\text { Previous Hx of NSVD at } 22 \text { wks, } \\
\text { preterm labor }\end{array}$ \\
\hline & $4 / 1$ & $\mathrm{COH}+\mathrm{IUI}$ & Uterine septum & 34 wks & R C/S Vx M/2210g, Vx M/2550g & Preterm labor \\
\hline & $3 / 0$ & IVF & Uterine septum & $30+5$ wks & $\mathrm{C} / \mathrm{S}$ Vx M/1350g, Br F/1140g & Preterm labor \\
\hline & $3 / 0$ & $\mathrm{COH}+\mathrm{IUI}$ & Uterine septum & $32+2$ wks & $\mathrm{C} / \mathrm{S} V \times \mathrm{M} / 1610 \mathrm{~g}, \mathrm{~V} \times \mathrm{F} / 900 \mathrm{~g}$ & Severe pre-eclampsia, preterm labor \\
\hline & $2 / 0$ & IVF & Uterine septum & 38 wks & $\begin{array}{l}\mathrm{C} / \mathrm{S} \text { placenta previa marginalis } \mathrm{Vx} \\
\mathrm{F} / 3000 \mathrm{~g}, \mathrm{Vx} \mathrm{F} / 2680 \mathrm{~g}\end{array}$ & Unremarkable \\
\hline & $1 / 0$ & IVF & Uterine septum & $36+6 \mathrm{wks}$ & $\mathrm{C} / \mathrm{S} \mathrm{Br} \mathrm{F/2880g}, \mathrm{V} \times \mathrm{M} / 2910 \mathrm{~g}$ & $\begin{array}{l}\text { Severe pre-eclampsia, postpartum } \\
\text { hemorrhage }\end{array}$ \\
\hline & $5 / 0$ & IVF & Uterine septum & $37+3 \mathrm{wks}$ & $\begin{array}{l}\mathrm{C} / \mathrm{S} \text { previous myomectomy, } \mathrm{Br} \\
\mathrm{M} / 3210 \mathrm{~g}, \mathrm{Br} \mathrm{F} / 2970 \mathrm{~g}\end{array}$ & $\begin{array}{l}\text { Multifetal reduction } 3->2 \text {, uterine } \\
\text { atony }\end{array}$ \\
\hline & $1 / 0$ & $\mathrm{COH}+\mathrm{IUI}$ & Uterine septum & $38+1$ wks & $\mathrm{C} / \mathrm{S}$ Vx F/2770g, F/3120g & Unremarkable \\
\hline & $1 / 0$ & $\mathrm{~T}-\mathrm{ET}$ & Uterine septum & $35+6$ wks & $\begin{array}{l}\text { C/S previous myomectomy } V x \\
\text { F/2020g, Br F/1660g }\end{array}$ & Severe pre-eclampsia \\
\hline
\end{tabular}

Note: a Only studies of twins delivered after 24 weeks of gestational age are included.

$\mathrm{G} / \mathrm{P}=$ gravida/parity; $\mathrm{IUI}=$ intrauterine insemination; wks $=$ weeks; $\mathrm{C} / \mathrm{S}=$ cesarean section; $\mathrm{Vx}=$ vertex; $\mathrm{Cx}=$ cervix; $\mathrm{PPROM}=$ preterm premature ruptures of membranes; $\mathrm{COH}=\mathrm{controlled}$ ovarian hyperstimulation; $\mathrm{Br}=$ breech; $\mathrm{T}-\mathrm{ET}=$ thawing-embryo transfer; $\mathrm{NSVD}=$ normal spontaneous vaginal delivery; $\mathrm{d} / \mathrm{t}=$ due to; $\mathrm{M}=$ male; $\mathrm{c}=$ with; $\mathrm{F}=$ female; $\mathrm{BP}=$ blood pressure; IVF-ET = in vitro fertilization-embryo transfer; IUGR $=$ intrauterine growth restriction; $\mathrm{CC}=$ clomiphene citrate; $\mathrm{R} \mathrm{C/S}=$ repeat cesarean section; $\mathrm{GDM}=$ gestational diabetes mellitus; $\mathrm{TI}=$ timed intercourse; $\mathrm{Hx}=$ history 
$.05)$, preterm birth $(p<.01)$, and lower birth weights $(p<.05)$.

Interestingly, our study and the Fox et al. (2014) study had the same number of cases, and furthermore, the types of MA were very similar: Fox et al. had 9 cases with septated uterus, 3 cases with bicornuate uterus, 3 cases with arcuate uterus, 1 case with unicornuate uterus, and 1 case with didelphys cases, and ours had 10 cases with septated uterus, 3 cases with bicornuate uterus, 2 cases with arcuate uterus, and 2 cases with unicornuate uterus cases. Originally, 34 cases of twin pregnancies with MA were found in our hospital during the study period. However, 11 of these cases, all of whom had septated uterus, were transferred to hospitals near the patients' homes because the patients visited our hospital only for the use of ART and wanted to give a birth in hospitals near their home. If we had included these 11 cases in our study, the results may have been different. However, we were not able to follow up these 11 cases.

Of the remaining 23 cases, except for 17 cases used in the present study, 6 cases had miscarriages before 24 gestational weeks (see the Appendix for a list of these cases). Of these 6 cases, 4 cases ( 1 bicornuate uterus and 3 septated uterus) had miscarriages (missed abortion) of both fetuses between 8 and 16 gestational weeks; and each of the remaining 2 cases (septated uterus) had miscarriages (missed abortion) of only one fetus, with the surviving fetuses being delivered at 41 weeks and 42 weeks, respectively. However, as the number of cases with MA and twin pregnancy that were terminated before 24 weeks was very small, it was difficult to determine causal factors that influenced mid-trimester loss versus survival. Future studies should focus on the effect of MA in the first and second trimesters of twin pregnancy to develop effective intervention strategies to improve the rate of fetal survival in early period of pregnancy.

Comparing baseline characteristics of the patients with MA and twin pregnancy between two studies, the rates of IVF pregnancy were higher in the Fox et al. (2014) study than in ours ( $76.5 \%$ vs. $64.7 \%)$, and while the Fox et al. study had one pair of monochorionic twins, ours had no monochorionic twins. Although both the Fox et al. study and ours were based on patients from a single hospital where patients are predominantly middle-class members, the patients in the Fox et al. study were mostly Caucasian women, whereas ours were Koreans. These differences may have contributed to discrepancies in findings between two studies. However, one should note that because of low prevalence of MA in twin pregnancy, differences in a few cases can lead to different conclusions.

For pregnancy outcomes, the mean gestational weeks at birth and the mean birthweight of twins delivered after 24 gestational weeks in patients with MA were higher in our study than in the Fox et al. study although the mean pre-pregnancy BMI of mothers with MA was almost identical and the types of anomalies were very similar in two studies. As there was no significant increase of fetal growth restriction in the MA group in the Fox et al. (2014) study, a significantly lower birthweight found by Fox et al. appeared to be a result of lower gestational weeks in their study. It may be that as compared to those in the Fox et al. study, our management strategies for women with MA and twin pregnancy were more effective in preventing preterm birth. In our hospital, in addition to routine examinations of blood pressure and maternal weight, urine tests, and monthly ultrasound measurement of fetal weight and amniotic fluid index, we put extra effort into providing regular education to our high-risk patients so that they understand the importance of preventing preterm birth and are able to detect possible signs and symptoms of preterm labor as early as possible. Whenever patients expressed any evidence of preterm labor or signals of the initiation of preterm labor, we performed uterine activity monitoring and ultrasonographic cervical assessment, and provided absolute bed rest, hydration, medication, and tocolysis if necessary. In our MA group, 10 patients delivered twins at term (Table 2). Two of these 10 patients visited our emergency room for the symptoms of preterm labor but were able to delay the delivery until the full term with our intervention. We think that our education programs about preterm labor helped the patients detect early signs of preterm labor and contributed to lowering number of cases of preterm delivery in our high-risk group.

Prior studies of MA on twin pregnancy were predominantly case reports. However, a case report is not informative in studying the effects of MA in twin pregnancy because they cannot distinguish whether or not adverse pregnancy outcomes were due to twin pregnancy or to MA. Thus, comparative studies with large samples are necessary to resolve the question of whether or not MA gives additional risks in twin pregnancy. Especially given the low prevalence of MA, collecting cases with international and/or domestic collaborations may be a good strategy to address this research issue.

There were several strengths and limitations in the present study. First, although our study is one of the largest in the literature of twin pregnancy with MA, the number of patients with MA and twin pregnancy in our study was still too small to make a strong conclusion. Thus, our findings should be interpreted with a caution and need to be replicated in other large samples. Second, our study did not divide MA by specific subtype due to the small number of patients. Future studies should increase the sample size and evaluate the effects of MA at the subtype level for a deeper understanding of the association between MA and twin pregnancy. Third, a large control group is a strength of our study as we were able to compare the rates of obstetrical complications such as pre-eclampsia and GDM, in addition to pregnancy outcomes between the patient and control group, while Fox et al. (2014) only compared pregnancy outcomes that focused mostly on fetal growth and delivery. Fourth, as we managed all twin pregnancies and MA with a uniform protocol in our hospital, we could 
control possible confounding variables arising from different treatment protocols across different hospitals. Using a discrete classification scheme, we also thoroughly reviewed each case in the twin database in our hospital to ensure accuracy of diagnosis. Finally, our data may have a selection bias as they were derived from a single hospital located in a relatively wealthy area of Seoul, Korea. As mentioned above, we were not able to include 11 cases from small towns in our analysis, which may limit generalizability of our findings to middle-class women in Seoul. Additionally, our sample was based on Korean women only. Given racial/ethnic differences in the mean level of birthweight (Hur et al., 2005), the impact of GDM in fetal growth (Hunt et al., 2012), and the prevalence and severity of obstetrical complications such as pre-eclampsia (Ghosh et al., 2014), our findings may not generalize to other ethnic/racial groups.

In conclusion, our study suggests that the presence of a congenital MA may not significantly increase risks on pregnancy outcomes and obstetrical complications in Korean women with twin pregnancy after 24 gestational weeks. Given the recent advance of ART and subsequent increase of twin pregnancies with MA, we think our data may be useful when clinicians counsel patients with MA who are at an increased risk of twin pregnancy.

\section{References}

Acien, P. (1993). Reproductive performance of women with uterine malformations. Human Reproduction, 8, 122-126.

Ahmad, F. K., Sherman, S. J., \& Hagglund, K. H. (2000). Twin gestation in a woman with a uterus didelphys. A case report. Journal of Reproductive Medicine, 45, 357-359.

American Fertility Society. (1988). The american fertility society classifications of adnexal adhesions, distal tubal occlusion, tubal occlusion secondary to tubal ligation, tubal pregnancies, Müllerian anomalies and intrauterine adhesions. (1988). Fertility and Sterility, 49, 944-955.

Arora, M., Gupta, N., \& Jindal, S. (2007). Unique case of successful twin pregnancy after spontaneous conception in a patient with uterus bicornis unicollis. Archives of Gynecology and Obstetrics, 276, 193-195.

Aruh, I., Aslan, D., Karaarslan, F., Pinar, T., Keles, R., \& Demir, N. (2005). Twin pregnancy in the horns of a bicornuate uterus after in vitro fertilization and split embryo transfer: A case report. Journal of Reproductive Medicine, 50, 213215.

Braun, P., Grau, F. V., Pons, R. M., \& Enguix, D. P. (2005). Is hysterosalpingography able to diagnose all uterine malformations correctly? A retrospective study. European Journal of Radiology, 53, 274-279.

Brown, O. (1999). Twin pregnancy in a uterus didelphys, with unilateral placental abruption and onset of labour. The Australian and New Zealand Journal of Obstetrics and Gynaecology, 39, 506-508.

Chan, Y. Y., Jayaprakasan, K., Tan, A., Thornton, J. G., Coomarasamy, A., \& Raine-Fenning, N. J. (2011). Reproductive outcomes in women with congenital uterine anomalies: A systematic review. Ultrasound in Obstetrics \& Gynecology, 38, 371-382.

Check, J. H., Lee, G., Nowroozi, K., \& Baker, A. (1992). Successful delivery of twins in a woman with a unicornuate uterus. Gynecology and Obstetric Investigation, 34, 124-125.

Cruceyra, M., Iglesias, C., De La Calle, M., Sancha, M., Magallon, S. L., \& Gonzalez, A. (2011). Successful delivery of a twin pregnancy in a bicornuate uterus (uterus bicornis unicollis) by bilateral Caesarean section. Journal of Obstetrics and Gynaecology Canada, 33, 142-144.

Doruk, A., Gozukara, I., Burkas, G., Bilik, E., \& Dilek, T. U. 365 (2013). Spontaneous twin pregnancy in uterus bicornis unicollis. Case Reports in Obstetrics and Gynecology, (2013) Article ID 834952. http://dx.doi.org/10.1155/2013/834952

Fox, N. S., Roman, A. S., Saltzman, D. H., Klauser, C. K., \& Rebarber, A. (2014). Twin pregnancy in patients with a uterine anomaly. The Journal of Maternal-Fetal Neonatal Medicine, 27, 360-364.

Ghosh, G., Grewal, J., Männistö, T., Mendola, P., Chen, Z, Xie, Y., \& Laughon, K. S. (2014). Racial/ethnic differences in pregnancy-related hypertensive disease in nulliparous women. Ethnicity \& Disease, 24, 283-289.

Hunt, K. J., Marlow, N. M., Gebregziabher, M., Ellerbe, C. N., Mauldin, J., Mayorga, M. E., \& Korte, J. E. (2012). Impact of maternal diabetes on birthweight is greater in non-Hispanic blacks than in non-Hispanic whites. Diabetologia, 55, 971980.

Hur, Y.-M., \& Kwon, J. S. (2005). Changes in twining rates in South Korea: 1981-2002. Twin Research and Human Genetics, 8, 76-79.

Hur, Y.-M., Luciano, M., Martin, N. G., Boomsma, D. I., Iacono, W. G., McGue, M., ... Han, J. Y. (2005). A comparison of twin birthweight data from Australia, the Netherlands, the United States, Japan, and South Korea: Are genetic and environmental variations in birthweight similar in Caucasians and East Asians?. Twin Research and Human Genetics, 8, 638-648.

Hur, Y-M., \& Song, T. B. (2009). A recent rise in twin birth rates and demographic changes in mothers of twins in South Korea: 2003-2007. Twin Research and Human Genetics, 12, 118-122.

Kekkonen, R., Nuutila, M., \& Laatikainen, T. (1991). Twin pregnancy with a fetus in each half of a uterus didelphys. Acta Obstetrica et Gynecologica Scandinavica, 70, 373-374.

Kim, H. J., Shin, H. M., Song, J. Y., Kim, S. Y., Chung, J. E., Kwon, D. J., ... Kim, M. R. (2008). Clinical characteristics of 110 women with uterine anomalies. Korean Journal of Obstetrics and Gynecology, 51, 1472-1480.

Kogan, M. D., Alexander, G. R., Kotelchuck, M., MacDorman, M. F., Buekens, P., \& Papiernik, E. (2002). A comparison of risk factors for twin preterm birth in the United States between 1981-82 and 1996-97. Maternal and Child Health Journal, 6, 29-35.

Krotz, S., Fajardo, J., Ghandi, S., Patel, A., \& Keith, L. G. (2002). Hypertensive disease in twin pregnancies: A review. Twin Research, 5, 8-14. 
Leible, S., Munoz, H., Walton, R., Sabaj, V., Cumsille, F., \& Sepulveda, W. (1998). Uterine artery blood flow velocity waveforms in pregnant women with Müllerian duct anomaly: A biologic model for uteroplacental insufficiency. American Journal of Obstetrics and Gynecology, 178, 10481053.

Lin, P. C., Bhatnagar, K. P., Nettleton, G. S., \& Nakajima, S. T. (2002). Female genital anomalies affecting reproduction. Fertility and Sterility, 78, 899-915.

Martin, J. A., Hamilton, B. E., Osterman, M. J., Curtin, S. C., \& Matthews, T. J. (2015). Births: Final data for 2013. National Vital Statistics Reports, 64, 1-65.

Nahra-Lynch, M., \& Toffle, R. C. (1997). Multiple gestation in a unicornuate uterus. A case report. Journal of Reproductive Medicine, 42, 451-454.

Nahum, G. G. (1997). Rudimentary uterine horn pregnancy. A case report on surviving twins delivered eight days apart. Journal of Reproductive Medicine, 42, 525-532.

Nanda, S., Dahiya, K., Sharma, N., Aggarwal, D., Sighal, S. R., \& Sangwan, N. (2009). Successful twin pregnancy in a unicornuate uterus with one fetus in the non-communicating rudimentary horn. Archives of Gynecology and Obstetrics, 280, 993-995.
Nohara, M., Nakayama, M., Masamoto, H., Nakazato, K., Sakumoto, K., \& Kanazawa, K. (2003). Twin pregnancy in each half of a uterus didelphys with a delivery interval of 66 days. BJOG, 110, 331-332.

Propst, A. M., \& Hill, J. A. (2000). Anatomic factors associated with recurrent pregnancy loss. Seminars in Reproductive Medicine, 18, 341-350.

Simon, C., Martinez, L., Pardo, F., Tortajada, M., \& Pellicer, A. (1991). Müllerian defects in women with normal reproductive outcome. Fertility and Sterility, 56, 1192-1193.

Sugaya, S. (2010). Twin pregnancy after in vitro fertilization in a woman with a unicornuate uterus. Clinical and Experimental Obstetrics \& Gynecology, 37, 317-318.

Weissman, A., Eldar, I., Malinger, G., Sadan, O., Glezerman, M., \& Levran, D. (2006). Successful twin pregnancy in a patient with complete uterine septum corrected during cesarean section. Fertility and Sterility, 85, 494, e1114.

Yarkoni, S., Reece, E. A., Holford, T., O’Connor, T. Z., \& Hobbins, J. C. (1987). Estimated fetal weight in the evaluation of growth in twin gestations: A prospective longitudinal study. Obstetrics and Gynecology, 69, 636639.

\section{APPENDIX}

Characteristics and Outcomes of Pregnancies With Müllerian Anomalies Terminated Before 24 Weeks of Gestation

\begin{tabular}{|c|c|c|c|c|c|c|c|}
\hline $\begin{array}{l}\text { Patient } \\
\text { num- } \\
\text { ber }\end{array}$ & Type of MA & $\mathrm{G} / \mathrm{P}$ & $\begin{array}{l}\text { Mode of } \\
\text { conception }\end{array}$ & $\begin{array}{l}\text { Delivery in } \\
\text { weeks }\end{array}$ & $\begin{array}{l}\text { Delivery } \\
\text { mode }\end{array}$ & $\begin{array}{l}\text { Fetal } \\
\text { presentation }\end{array}$ & Others \\
\hline 1 & Bicornuate uterus & $2 / 0$ & IVF & $9+6$ & $\mathrm{D} / \mathrm{E}$ & & Missed abortion \\
\hline 2 & Uterine septum & $3 / 1$ & IVF & $6+6$ & $D / E$ & & Missed abortion \\
\hline 3 & Uterine septum & $2 / 0$ & IVF & 8 & $D / E$ & & Missed abortion \\
\hline 4 & Uterine septum & $1 / 0$ & IVF & 8 & $\mathrm{D} / \mathrm{E}$ & & Missed abortion \\
\hline 5 & Uterine septum & $0 / 0$ & IVF & 41 & $\mathrm{C} / \mathrm{S}$ & $\mathrm{Br}$ & $\begin{array}{l}\text { Twin pregnancy } \rightarrow \text { one missed } \\
\text { abortion at } 9+4 \text { wks; Singleton } \\
\text { delivery at term }\end{array}$ \\
\hline 6 & Uterine septum & $2 / 0$ & Natural & 42 & $\mathrm{C} / \mathrm{S}$ & $\mathrm{Vx}$ & $\begin{array}{l}\text { Twin pregnancy } \rightarrow \text { one missed } \\
\text { abortion at } 11 \mathrm{wks}, \mathrm{C} / \mathrm{S} \mathrm{d} / \mathrm{t} \\
\text { progress failure; Singleton } \\
\text { delivery at term }\end{array}$ \\
\hline
\end{tabular}

Note: $\mathrm{G} / \mathrm{P}=$ gravida/parity; IVF $=$ in vitro fertilization; $\mathrm{D} / \mathrm{E}=$ dilatation and evacuation; $\mathrm{C} / \mathrm{S}=$ cesarean section; $\mathrm{Br}=\mathrm{breech} ; \mathrm{wks}=$ weeks; $\mathrm{Vx}=\mathrm{vertex} ; \mathrm{d} / \mathrm{t}=$ due to. 